\title{
Being preterm and small for gestational age had different effects on cognitive and motor development
}

\author{
Hutton JL, Pharoah PO, Cooke RW, et al. Differential effects of preterm birth and small gestational age on cognitive and motor \\ development. Arch Dis Child 1997 Mar;76:F75-81.
}

\section{Objective}

To determine the differential effects of preterm birth and being small for gestational age on cognitive and motor development of children.

\section{Design}

Inception cohort followed up until aged 8-9 years.

\section{Setting}

2 counties in the UK.

\section{Patients}

All children born in 1980 and 1981 who were $\leqslant 32$ weeks gestation and weighed $\leqslant 2000 \mathrm{~g}$. 182 children were identified and 158 ( $55 \%$ boys, $18 \%$ twins or triplets) took part in the study. Children with major disabilities and congenital abnormalities were excluded (cerebral palsy, Down's syndrome, blindness, and epilepsy).

\section{Assessment of prognostic factors}

Gestational age was based on the date of the mother's last menstrual period and clinical assessment. Small for gestational age was assessed using the gestational age and the expected birth weight (ratio of actual birth weight to expected birth weight). Smoking history of the mother during pregnancy and socioeconomic factors were also obtained (number of state benefits received, living in owner occupied housing, and educational achievements of the mother).

\section{Main outcome measures}

The Wechsler Intelligence Scale for Children was used to assess overall intellectual capacity (IQ) for speech and performance.
The Neale analysis of reading ability and the Stott-MoyesHenderson test of motor impairment were done.

\begin{abstract}
Main results
In a multivariate analysis, higher overall IQ was associated with a higher birth weight ratio, $\leqslant 1$ state benefit, the mother being in school after age 16, and house ownership. Higher verbal IQ was associated with the same variables and higher performance IQ was associated with a higher birth weight ratio and house ownership. Motor impairment was associated with a lower birth weight ratio, higher birth weight, and younger gestational age. The measures of reading ability were not associated with any study variables although subgroup analyses showed some associations: girls had better reading accuracy and reading rates; those receiving $\geqslant 1$ state benefit showed poorer reading rates, accuracy, and comprehension; reading accuracy and comprehension were lower if the mother had left school by age 16; and comprehension was greater for children living in owned houses.
\end{abstract}

\section{Conclusions}

Cognitive development was associated with being small for gestational age; motor development was additionally associated with preterm birth. Reading was affected more by social factors than birth factors.

\section{Source of funding: Department of Health.}

For article reprint: Professor P O Pharoah, Department of Public Health, Whelan Building Quadrangle, University of Liverpool, Liverpool, L69 3BX, UK. Fax +44 (0)151 7945588.

\section{Commentary}

Limited research is available on developmental outcomes of preterm, small for gestational age infants. The study by Hutton $e t a l$ is one of the few studies that considered birth weight within the same levels of maturity, therefore looking at the differential effects of being premature and being growth restricted.

The participants were all from 2 counties in the UK and so the findings may differ for other populations. Growth restriction was defined by birth weight, and did not incorporate a measure of head circumference or linear skeletal growth. Both of these variables are important to consider in growth restriction because they indicate different causative factors which might influence the long term outcomes. The authors caution that the findings cannot be extrapolated to infants $>32$ weeks gestational age who are small for gestation.
The results of this study are relevant to neonatal nurses, nurses who work in neonatal follow up or growth and development clinics, paediatric nurses, and public health nurses whose clientele include infants and children. The study is also relevant to neonatal nurse practitioners and neonatal and developmental care clinical nurse specialists. The findings of this study increase our knowledge of factors that affect outcomes of preterm and small for gestation infants. The results reinforce the importance of social factors and their effect on long term outcomes, such as IQ and reading ability. This knowledge is useful to nurses in their efforts to identify infants and parents at risk so that appropriate and timely supports can be mobilised.

The study results emphasise the difference between cognitive and motor abilities.
For advanced practitioners who discuss an infant's prognosis with parents, the findings reinforce the need to provide differential information on long term outcomes, such as reading and motor and cognitive abilities rather than discussing developmental outcomes in general. Although birth weight ratio, birth weight, and gestational age predicted cognitive and motor abilities in preterm and small for gestation infants, other variables added considerable explanatory power to their overall effects.

Janet Pinelli, RN, DNS

Clinical Nurse Specialist/ Neonatal Practitioner Neonatal Intensive Care Unit Hamilton Health Sciences Corporation Hamilton, Ontario

Canada 